\title{
Fish Collagen Promotes the Expression of Genes Related to Osteoblastic Activity
}

\author{
Mark Luigi Fabian Capati, Ayako Nakazono, Kohei Yamamoto, Kouji Sugimoto, \\ Kajiro Yanagiguchi, Shizuka Yamada, and Yoshihiko Hayashi \\ Department of Cariology, Nagasaki University Graduate School of Biomedical Sciences, Nagasaki 852-8588, Japan \\ Correspondence should be addressed to Yoshihiko Hayashi; hayashi@nagasaki-u.ac.jp
}

Received 5 July 2016; Accepted 25 August 2016

Academic Editor: Yin Chen

Copyright ( 2016 Mark Luigi Fabian Capati et al. This is an open access article distributed under the Creative Commons Attribution License, which permits unrestricted use, distribution, and reproduction in any medium, provided the original work is properly cited.

\begin{abstract}
Tilapia type I atelocollagen (TAC) is a strong candidate for clinical application as its biological scaffold due to a high degeneration temperature and biologically safe properties. The aim of this study was to confirm the biological effects of TAC in vitro on osteoblastic cells, simulating its clinical application. The proliferation and differentiation of typical preosteoblasts, MC3T3-E1 cells, were investigated using a microarray analysis, staining assay for mineralization, and real-time PCR analysis of the expression of mineralization-related genes. The mRNA expression of 10 genes involved in proliferation and differentiation increased after 3-day culture on an TAC gel, with an average balanced score ratio exceeding 1.5 compared to the control. After two weeks of culture, all three experimental groups showed stronger alkaline phosphatase staining than after one week. The genes expression of alkaline phosphatase, osteocalcin, and bone sialoprotein increased under the experimental conditions. The gene expression of osteopontin did not increase, and no statistical differences were noted among the three experimental groups. The present and previous findings suggest that TAC is not only a suitable alternative to collagen products originating from mammals but also a novel biomaterial with cell differentiation ability for regenerative medicine.
\end{abstract}

\section{Introduction}

The research and development of nonmammalian natural biomaterials will improve the safety of scaffold and carrier in regenerative medicine, which are meaningful and beneficial, due to the global prevalence of dangerous infectious diseases (zoonosis) such as bovine spongiform encephalopathy, avian and swine influenza, tooth-and-mouth disease in cows, pigs, and buffalos, Ebola hemorrhagic fever, and Zika fever [1]. Furthermore, collagen generated from porcine and bovine sources cannot be used due to religious objections in some countries. Fish, which are the most genetically distant relatives from mammals, have recently attracted attention as an alternative source of collagen. Fish collagen (FC) derived from the scales, skin, and bone has interested many laboratories worldwide for its bioactive properties, such as excellent biocompatibility, low antigenicity, high biodegradability, and cell proliferation potential $[2,3]$.
The weakest point of fish collagen is its relatively low denaturation temperature $\left(T_{d}\right)$, which indicates a poor stability in clinical applications. However, our recent experiment using calorimetry revealed that $T_{d}$ of collagen solution derived from the tropical fish, tilapia (tilapia type I atelocollagen $[\mathrm{TAC}]$ ), was $35-36^{\circ} \mathrm{C}[4]$, indicating that the abovementioned weak point has been completely overcome. In addition, the biological safety of TAC has been confirmed using various test methods recommended with ISO standards [5]. The long-term storage of TCA under frozen conditions for over 1 year has already been demonstrated in our laboratory (unpublished data). These data and findings strongly support TAC as a candidate for clinical application as a biological scaffold.

In the present experiment, we confirmed the biological effects of TAC in vitro on osteoblastic cells simulating its clinical application as a suitable alternative to collagen products 
TABLE 1: Primers designed for RT-PCR analyses of genes related to cell proliferation and differentiation.

\begin{tabular}{|c|c|}
\hline Gene name & Oligonucleotides \\
\hline \multirow{2}{*}{ Matrix metallopeptidase 13 (Mmp13) } & Forward: AGGCCTTCAGAAAAGCCTTC \\
\hline & Reverse: GGTCCTTGGAGTGATCCAGA \\
\hline \multirow{2}{*}{ Wnt inhibitory factor 1 (Wifl) } & Forward: GAGTGTCCGGATGGGTTCTA \\
\hline & Reverse: TGGTTGAGCAGTTTGCTTTG \\
\hline \multirow{2}{*}{ Receptor activity modifying protein 1 (Ramp1) } & Forward: GCGGTATCCTCCTGAAAACA \\
\hline & Reverse: CAGTCCTCCAGTTGGACCAT \\
\hline \multirow{2}{*}{ SMAD family member 6 (Smad6) } & Forward: ACGGTGACCTGCTGTCTCTT \\
\hline & Reverse: AGCGAGTACGTGACCGTCTT \\
\hline \multirow{2}{*}{ Platelet-derived growth factor, D polypeptide (Pdgfd) } & Forward: TCAGCTGTGTGCTCAACAAA \\
\hline & Reverse: ATTGGGCCTGGCTTACTTCT \\
\hline \multirow{2}{*}{ Chordin-like 1 (Chrd1) } & Forward: TGGTCTTTGCTTTCCCATGT \\
\hline & Reverse: CCCAGGTGTTCTCTGAAAGC \\
\hline \multirow{2}{*}{ Septin 4 (Sept4) } & Forward: TTCAGGTCCAAAAGCCAGTT \\
\hline & Reverse: TGACTTCCCTATCCCTGCTG \\
\hline \multirow{2}{*}{ Fibroblast growth factor receptor 3 (Fgfr3) } & Forward: ACAAGGACCGTACTGCCAAG \\
\hline & Reverse: ACCTGGTAGGCACAGGACAC \\
\hline \multirow{2}{*}{ Lumican (Lum) } & Forward: TTCTCTCTTGCCTTGGCATT \\
\hline & Reverse: CACTGCAGGTCTGTGACGTT \\
\hline \multirow{2}{*}{ Vitamin D receptor $(\mathrm{Vdr})$} & Forward: CGGAAATGGGTACCAAAATG \\
\hline & Reverse: CACGTAGCAAGCGCTATGAA \\
\hline \multirow{2}{*}{ Ribosomal protein S17 (rpS17) } & Forward: GCATATCATGCAACGCTTTC \\
\hline & Reverse: GGAGCTTCAGCATCTCCTTG \\
\hline
\end{tabular}

originating from mammals. The proliferation and differentiation of MC3T3-E1 cells, which are typical preosteoblasts, were investigated using a microarray analysis, staining assay for mineralization, and real-time PCR analysis (RT-PCR) to determine the expression of calcification-related genes.

\section{Materials and Methods}

2.1. Cell Culture for Passage. The used MC3T3-E1 cells derived from mouse calvaria were the typical preosteoblasts. They were cultured in a $100 \mathrm{~mm}$ dish at a density of $1.5 \times$ $10^{6}$ cells in $\alpha$-MEM (Gibco, Palo Alto, CA, USA) with $10 \%$ fetal bovine serum (FBS) (MP Biomedicals, Santa Ana, CA, USA) and penicillin-streptomycin (Life Technologies, Carlsbad, CA, USA) and then incubated [6].

2.2. The Dissolution of TAC. TAC (0.6\%) produced by solubilized tilapia skin was supplied by Nippi Inc., Biomatrix Institute (Ibaraki, Japan). A diluted $0.1 \%$ TAC solution in 1.5-fold concentration PBS (-) $(\mathrm{pH} 7.4)$ was used for the biological experiments.

2.3. Total RNA Isolation for Microarray. For FC gel group, $1 \mathrm{~mL}$ of $0.1 \%$ TAC was added to a $60 \mathrm{~mm}$ culture dish. TCA solution was gelled at $37^{\circ} \mathrm{C}$ for $30 \mathrm{~min}$ in a condition of $5 \%$ $\mathrm{CO}_{2}$ and air. MC3T3-E1 cells were seeded in a $60 \mathrm{~mm}$ culture dish covered with or without TAC gel at a density of $1.5 \times$ $10^{6}$ cells. After 3 -day culture, total RNA from cells was isolated using TRIzol reagent $\left(1 \mathrm{~mL} / 10 \mathrm{~cm}^{2}\right)$ (Invitrogen, Carlsbad, CA, USA) and purified in accordance with the manufacturer's instructions [6].

2.4. Microarray of Gene Expression for Checking Proliferation and Differentiation [6]. The cRNA was amplified and hybridized to an Affinity Mouse Genome 4302.0 array (for 39,000 transcriptional products), scanned by an Affymetrix scanner, and calculated using the Affymetrix Expression Console $^{\mathrm{TM}}$.

2.5. Filter Criteria for Data Analysis [6]. We then established the criteria for upregulated genes $(Z$ score $>3.0$, ratio $>1.5$ fold).

2.6. RT-PCR following Microarray. The MC3T3-E1 cells were seeded and cultured ( $n=3$ for FC group, $n=3$ for control group) under the same conditions as in the experiment for total RNA isolation for microarray. The primers are indicated in Table 1. The analyses of RT-PCR were carried out according to the similar methods to our recent study [6], except the following two steps. The first was that the protocol of amplification and quantification was 40 cycles $\left(58-59^{\circ} \mathrm{C}\right.$, $1 \mathrm{~min})$. The second was that, in the final calculations, the data were normalized by ribosomal protein S17.

2.7. Alkaline Phosphatase (ALP) Staining. For the FC gel group, $90 \mu \mathrm{L}$ of $0.1 \%$ TAC was poured into each well. TCA solution was gelled at $37^{\circ} \mathrm{C}$ for $30 \mathrm{~min}$ in an atmosphere of 
TABLE 2: Primers designed for RT-PCR analyses of gene related to calcification.

\begin{tabular}{ll}
\hline Gene name & Oligonucleotides \\
\hline Alkaline phosphatase (ALP) & Forward: GTTGCCAAGCTGGGAAGAACAC \\
Osteopontin (OPN) & Reverse: CCCACCCCGCTATTCCAAAC \\
Osteocalcin (OCN) & Forward: CATTGCCTCCTCCCTCCCGGTG \\
Bone sialoprotein (BSP) & Reverse: GCTATCACCTCGGCCGTTGGGG \\
Ribosomal protein S17 (rpS17) & Forward: AGGGAGGATCAAGTCCCG \\
& Reverse: GAACAGACTCCGGCGCTA \\
& Forward: TGTCTGCTGAAACCCTTC \\
& Reverse: GGGGTCTTTAAGTACCGGC \\
& Forward: GCATATCATGCAACGCTTTC \\
\end{tabular}

$5 \% \mathrm{CO}_{2}$ and air. The MC3T3-E1 cells were cultured in a 24well plate at a density of $5 \times 10^{4}$ cells/well and incubated for 1 or 2 weeks. The following four groups $(n=3$ wells for each group and each week) were designed for comparison: (1) the control group (culture medium: $\alpha$-MEM with FBS), (2) FC-positive group (culture medium: $\alpha$-MEM with FBS), (3) FC-negative and BGP-positive group \{culture medium: $\alpha$-MEM supplemented with FBS, $50 \mu \mathrm{g} / \mathrm{mL}$ ascorbic acid (Wako, LTD., Osaka, Japan), and $10 \mathrm{mM} \beta$-glycerophosphate ([BGP] nakarai tesque, Kyoto, Japan)\}, (4) FC-positive and BGP-positive group (culture medium: $\alpha$-MEM with FBS, $50 \mu \mathrm{g} / \mathrm{mL}$ ascorbic acid, and $10 \mathrm{mM}$ BGP). The medium was renewed every three days. The ALP Staining Kit (Stemgent, Inc., Cambridge, MA, USA) was used to stain for ALP expression in accordance with the maker's instructions.

2.8. RT-PCR for Calcification-Related Genes. For the FC gel group, $1 \mathrm{~mL}$ of $0.1 \%$ TAC was added to a $60 \mathrm{~mm}$ culture dish. TCA solution was gelled at $37^{\circ} \mathrm{C}$ for $30 \mathrm{~min}$ in a condition of $5 \% \mathrm{CO}_{2}$ and air. MC3T3-E1 cells were seeded in a $60 \mathrm{~mm}$ culture dish covered with or without TAC gel at a density of $1.5 \times 10^{6}$ cells. The abovementioned four groups were also prepared for this experiment ( $n=3$ for each group). After three days of culture, RT-PCR was applied to check the expression of four calcification-related genes: ALP, osteopontin (OPN), osteocalcin (OCN), and bone sialoprotein (BSP). All the methods were similar to the abovementioned methods. The primer designs for these genes are represented in Table 2.

2.9. Statistical Analyses. All data were represented as the mean \pm standard deviation (SD). The statistical significance $(P<0.05)$ of the differences between the two different experimental groups was demonstrated using a paired Student's $t$ test.

\section{Results}

3.1. Genes Expression to Proliferation and Differentiation. Microanalysis revealed 72 of the upregulated genes ( $Z$ score $>$ 3.0). The following 10 genes related to proliferation and differentiation of osteoblastic cells were compared with the control using the RT-PCR: vitamin D receptor (Vdr), lumican (Lum), matrix metallopeptidase 13 (Mmp13), SMAD family
TABLE 3: Genes expression represented in cell proliferation and differentiation.

\begin{tabular}{lccc}
\hline Gene name & $Z$ score & Ratio $^{\mathrm{a}}$ & Confirmation $^{\mathrm{b}}$ \\
\hline Vdr & 3.9 & 1.6 & 1.5 \\
Lum & 4.9 & 1.7 & 1.5 \\
Mmp13 & 12.2 & 4.4 & 3.5 \\
Smad6 & 3.7 & 1.5 & 2.0 \\
Pdgfd & 3.4 & 1.5 & 1.6 \\
Wif1 & 6.3 & 2.0 & 5.1 \\
Fgfr3 & 4.8 & 1.7 & 2.0 \\
Sept4 & 6.5 & 2.1 & 2.3 \\
Ramp1 & 4.3 & 1.6 & 3.2 \\
Chrdl1 & 6.0 & 1.9 & 3.9
\end{tabular}

${ }^{\mathrm{a}}$ The change raw signal ratio. ${ }^{\mathrm{b}}$ Confirmed by the RT-PCR data (triplicate samples).

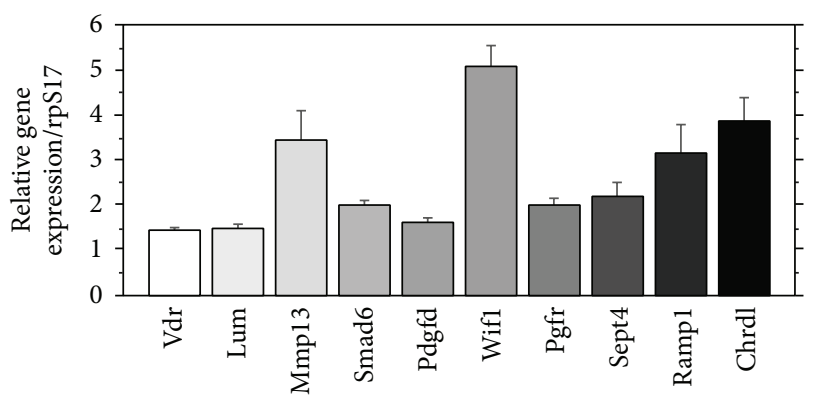

FIGURE 1: RT-PCR for 10 genes in relation to cell proliferation and differentiation. Each bar indicates the SD of triplicate samples.

member 6 (Smad6), platelet-derived growth factor D (Pdgfd), Wnt inhibitory factor 1 (Wif1), fibroblast growth factor receptor 3 (Fgfr3), septin 4 (Sept4), receptor (calcitonin) activity modifying protein 1 (Ramp1), and chordin-like 1 (Chrdl1) (Table 3). The RT-PCR analyses revealed that the mRNA expression of these 10 genes also increased after 3day culture on FC gel, with an average balanced score ratio exceeding 1.5 compared to the control (Figure 1).

3.2. Alkaline Phosphatase Staining (Figure 2). In group (1) after 2 weeks of culture, very-weak-intensity ALP staining 
1 week

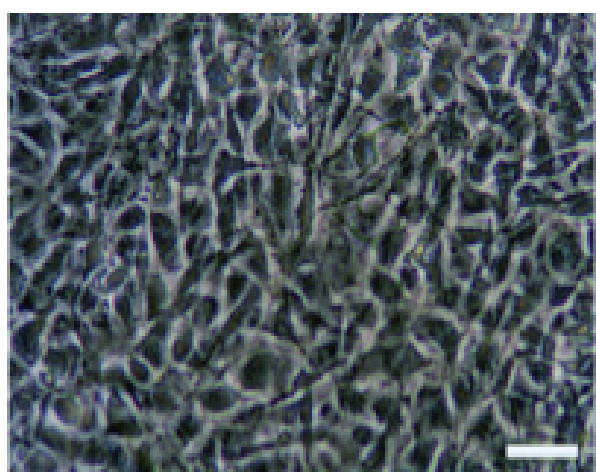

(a)

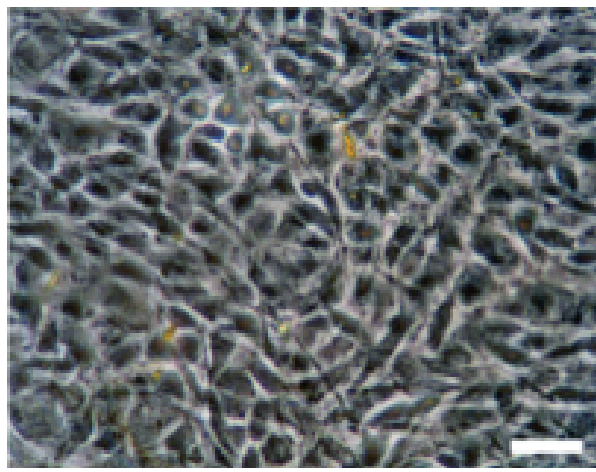

(b)

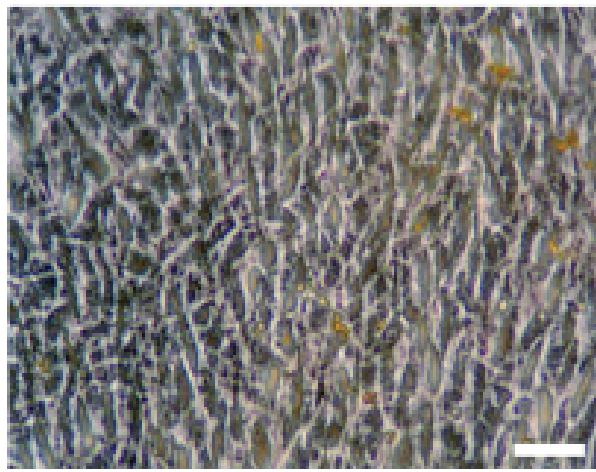

(c)

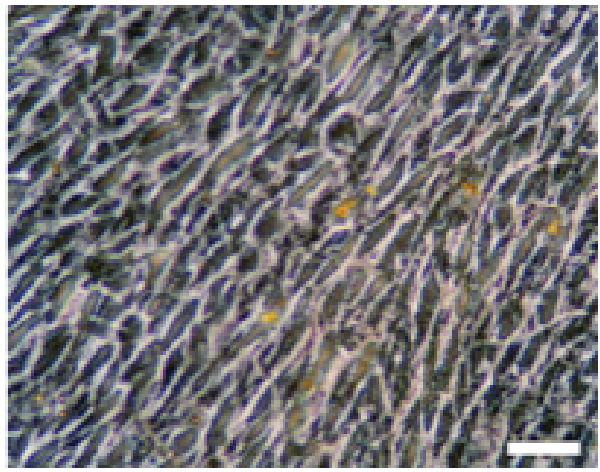

(d)
2 weeks

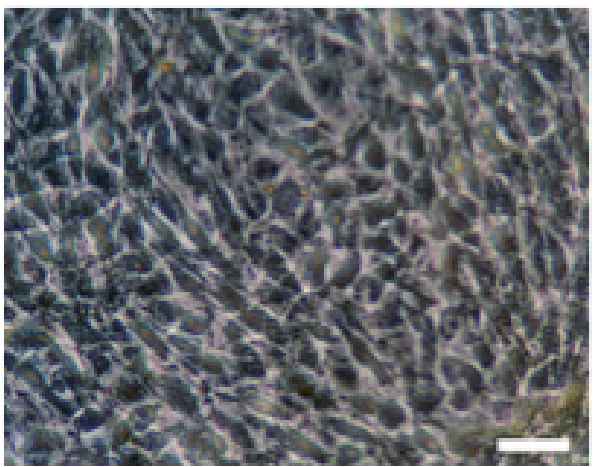

(e)

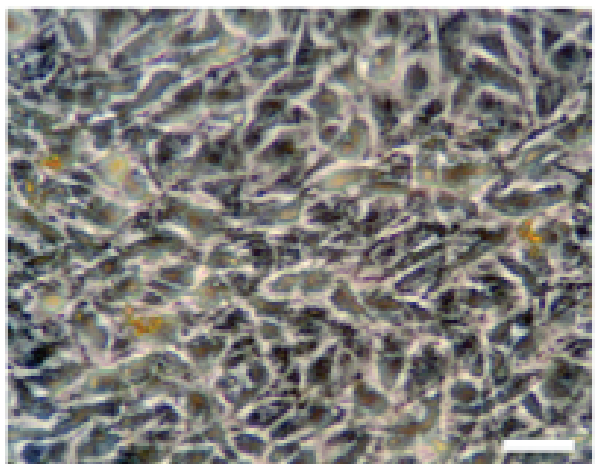

(f)

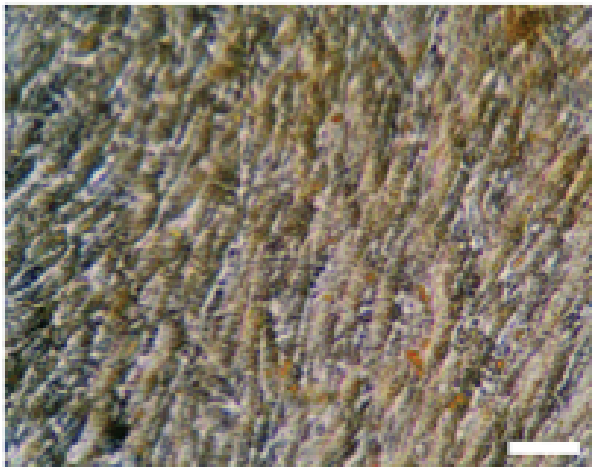

(g)

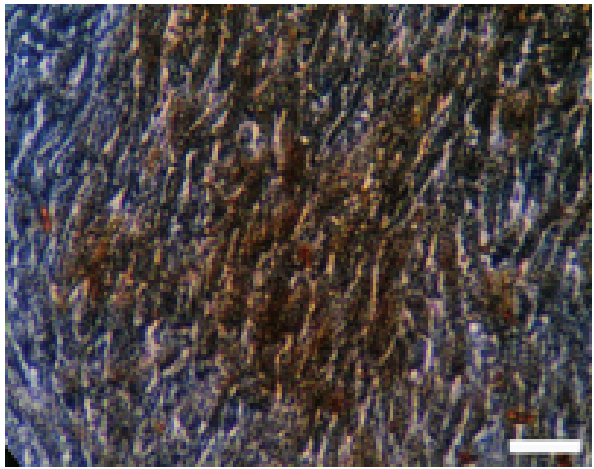

(h)

FIGURE 2: Representative ALP staining photographs after one or two weeks of culture: (a, e) FC-negative group, (b, f) FC-positive group, (c, g) FC-negative and BGP-positive group, and (d, h) FC-positive and BGP-positive group. ALP staining shows either a yellow or brown color. Although the typical pavement-like shape is observed at (a), (e), and (b), (f), on the whole, the cell morphology changes to a spindle-like cell shape (c, g, and d, h). Scale bar $=15 \mu \mathrm{m}$. 


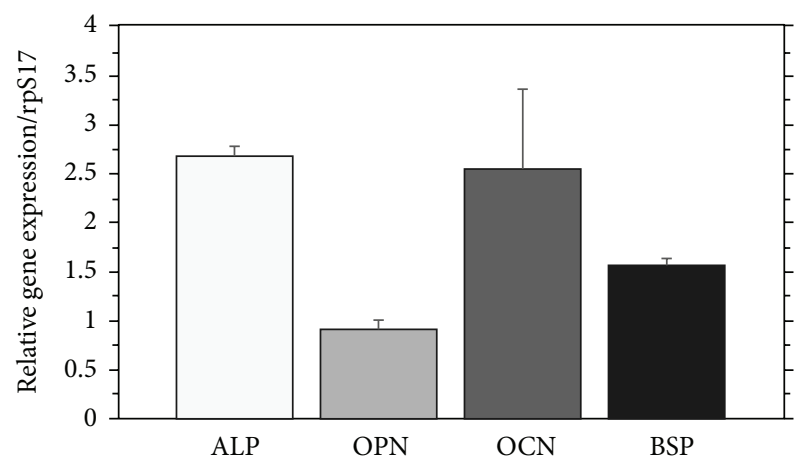

(a)

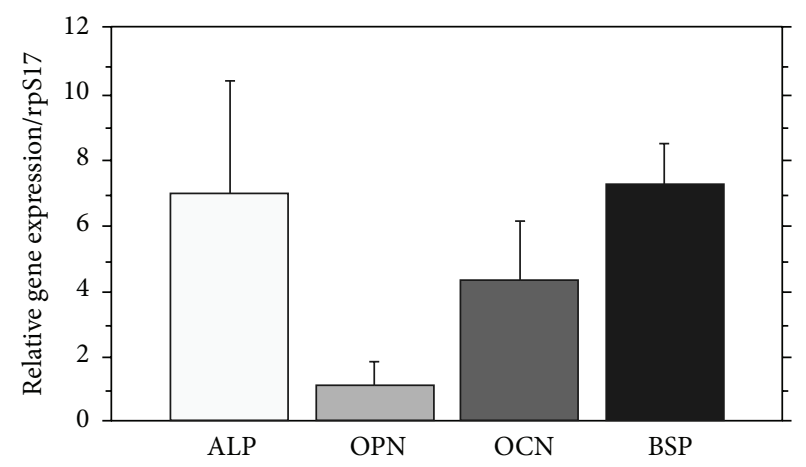

(b)

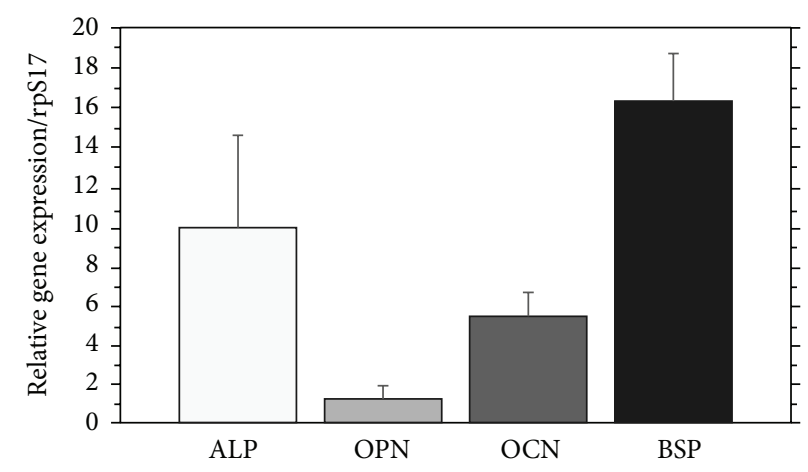

(c)

FIGURE 3: RT-PCR for four calcification-related genes: (a) FC-positive group (group 2), (b) FC-negative and BGP-positive group (group 3), and (c) FC-positive and BGP-positive group (group 4). Each bar represents the SD of triplicate samples.

was observed, similar to that in group (2) after 1 week. After 2 weeks of culture, each group showed stronger staining than after 1 week. On the whole, the spindle-like shape was observed in groups (3) and (4) (Figures 2(c), 2(g), and 2(d), $2(\mathrm{~h}))$. The intensity of staining increased with increasing group number from group (2) to group (4).

3.3. Gene Expression for Calcification. The RT-PCR analyses demonstrated that the mRNA expression of four calcification-related genes in three experimental groups was analyzed after 3-day culture, compared to the control group (1) (Figure 3). Although the gene expression of ALP, OCN, and BSP increased with increasing group number from group (2) to group (4), a significant increase was recognized in BSP gene expression $(P<0.01)$. The raw data of OPN expression of OPN was around 1.0 in three groups, although there was a significant increase between group (2) and group (3) $(P<$ $0.05)$.

\section{Discussion}

Cell culture method using the type I collagen gel was first reported by Elsdale and Bard [7]. The cells have well grown within a three-dimensional (3D) structure, as the conditions more closely resemble those of the in vivo condition of the osteoblast than the traditional two-dimensional (2D) cultures. Cells growth in 3D gels showed positive mineral staining and induction of osteoblastic marker genes earlier than cell growth in $2 \mathrm{D}$ cultures [8]. Although the present culture conditions were not the exact 3D gel culture system, the present environment was remarkably similar to a $3 \mathrm{D}$ culture environment, as a low-concentration TCA solution was gelled to mimic wet and elastic conditions [9].

Microarray and RT-PCR analyses for checking the genes involved in proliferation and differentiation in MC3T3-E1 cells demonstrated that the $Z$ score data using the standard deviations presented the effect of FC on MC3T3-E1 cells more clearly than the original ratio results. Ten genes, including Wifl, a secreted modulator of Wnt proteins [10], increased expressions compared with a control condition. This datum is the first to reveal that FC directly accelerates the activity of MC3T3-E1 cells in order to maintain the preosteoblast nature.

In the present experiment, the function of MC3T3-E1 cells was accelerated after three-day culture in FC-positive or BGP-positive group, which was confirmed by the increase of the expression of calcification-related genes, except OCN. ALP is an early expression marker essential for osteoblast proliferation [11], which was well demonstrated by the present ALP staining findings and the typical change in the cell shape. Furthermore, the time lapse between mRNA expression (3 days) and protein synthesis (7 days) seemed reasonable. Although OPN, OCN, and BSP are generally used as differentiation markers for osteoblasts [11-13], OPN was also reported to act as a mineralization inhibitor to regulate crystal growth $[14,15]$. The present PCR data of OPN clearly demonstrated this osteoclastic function of OPN. 
Although the intracellular signal transduction pathway and the related gene expression in osteoblast differentiation have been widely investigated [16-22], the direct trigger mechanisms for osteoblast proliferation and differentiation by collagen have not yet been clarified. Recently, an interesting study revealed that adhesion of human mesenchymal stem cells on tilapia collagen in vitro was faster and higher than that on porcine collagen or a noncoated surface, probably due to the texture flexibility and surface softness of the tilapia scale collagen, making the binding between collagen and integrin easier to trigger and stimulate both proliferation and differentiation of preosteoblasts [23]. A 0.1\% TAC solution was used to make a gel in the present FC group. The nature of this gel is very soft, even after 2 weeks in the $\mathrm{CO}_{2}$ incubator, and is thought to be possessed of sufficient flexibility and surface softness. This means that cultured cells may also behave as they would in a 3D culture system.

Proper biodegradation and suitable biocompatibility are indispensable for biomaterials. The biodegradation of collagen is closely linked to and dependent on the constitution of amino acids in a given species. Compared to bovine collagen, the contents of methionine, isoleucine, leucine, hydroxylysine, and lysine are lower in TAC [24]. As these amino acids are related to the collagen stiffness, the tilapia collagen can be easily degraded in vivo. The characteristics of these amino acids give TAC its abovementioned nature.

From our previous data $[4,5]$ and the present results, TAC may represent not only a promising alternative to mammalian and avian collagen products but also a novel biomaterial with cell differentiation ability. The final goal with TAC is its clinical application as a scaffold in regenerative medicine. The enzymatic digestion time for atelotreatment and the elimination of enzyme in TAC are controlled to a clinical level (personal communication). The preliminary experimental data concerning pulp regeneration [24] using TAC as a scaffold revealed no rejection reaction on the newly regenerated pulp tissue in dogs (unpublished data). These findings suggest that TAC may be useful for clinical application after further animal experiments.

\section{Conclusion}

The present in vitro experiments using osteoblasts clearly revealed that TAC is not only a suitable alternative to collagen products originating from mammals for cell proliferation and differentiation as the scaffold for regenerative medicine.

\section{Competing Interests}

The authors declare no conflicts of interests in association with this study.

\section{Authors' Contributions}

Mark Luigi Fabian Capati and Ayako Nakazono contributed equally to this work.

\section{Acknowledgments}

This study was supported in part by Funds for Integrated Promotion of Social System Reform and Research and Development, Contract Grant Sponsor: Ministry of Education, Science, Sports, and Culture of Japan.

\section{References}

[1] Y. Hayashi, S. Yamada, T. Ikeda, Z. Koyama, and K. Yanagiguchi, "Chitosan and fish collagen as biomaterials for regenerative medicine," in Marine Medical Food, S.-K. Kim, Ed., vol. 65, chapter 6, pp. 107-120, Academic Press, London, UK, 2012.

[2] A. K. Dillow and A. M. Lowman, Biomimetic Materials and Design: Biointerfacial Strategies, Tissue Engineering, and Targeted Drug Delivery, Mercel Dekker, New York, NY, USA, 2002.

[3] S. Yang, K.-F. Leong, Z. Du, and C.-K. Chua, "The design of scaffolds for use in tissue engineering. Part I. Traditional factors," Tissue Engineering, vol. 7, no. 6, pp. 679-689, 2001.

[4] K. Yamamoto, Y. Yoshizawa, K. Yanagiguchi, T. Ikeda, S. Yamada, and Y. Hayashi, "The characterization of fish (tilapia) collagen sponge as a biomaterial," International Journal of Polymer Science, vol. 2015, Article ID 957385, 5 pages, 2015.

[5] K. Yamamoto, K. Igawa, K. Sugimoto et al., "Biological safety of fish (tilapia) collagen," BioMed Research International, vol. 2014, Article ID 630757, 9 pages, 2014.

[6] M. L. F. Capati, A. Nakazono, K. Igawa et al., "Boron accelerates cultured osteoblastic cell activity through calcium flux," Biological Trace Element Research, 2016.

[7] T. Elsdale and J. Bard, "Collagen substrata for studies on cell behavior," Journal of Cell Biology, vol. 54, no. 3, pp. 626-637, 1972.

[8] B. G. Matthews, D. Naot, K. E. Callon et al., "Enhanced osteoblastogenesis in three-dimensional collagen gels," Bonekey Reports, vol. 3, article 560, 2014.

[9] E. Hesse, T. E. Hefferan, J. E. Tarara et al., "Collagen type I hydrogel allows migration, proliferation, and osteogenic differentiation of rat bone marrow stromal cells," Journal of Biomedical Materials Research Part A, vol. 94, no. 2, pp. 442449, 2010.

[10] S. W. Cho, J.-Y. Yang, H. J. Sun et al., "Wnt inhibitory factor (WIF)-1 inhibits osteoblastic differentiation in mouse embryonic mesenchymal cells," Bone, vol. 44, no. 6, pp. 1069-1077, 2009.

[11] T. A. Owen, M. Aronow, V. Shalhoub et al., "Progressive development of the rat osteoblast phenotype in vitro: reciprocal relationships in expression of genes associated with osteoblast proliferation and differentiation during formation of the bone extracellular matrix," Journal of Cellular Physiology, vol. 143, no. 3, pp. 420-430, 1990.

[12] J. E. Aubin, F. Liu, L. Malaval, and A. K. Gupta, "Osteoblast and chondroblast differentiation," Bone, vol. 17, no. 2, supplement 1, pp. S77-S83, 1995.

[13] J. B. Lian and G. S. Stein, "Development of the osteoblast phenotype: molecular mechanisms mediating osteoblast growth and differentiation," The Iowa Orthopaedic Journal, vol. 15, pp. 118140, 1995.

[14] H. Yoshitake, S. R. Rittling, D. T. Denhardt, and M. Noda, "Osteopontin-deficient mice are resistant to ovariectomyinduced bone resorption," Proceedings of the National Academy of Sciences of the United States of America, vol. 96, no. 14, pp. 8156-8160, 1999. 
[15] J. Sodek, B. Ganss, and M. D. McKee, “Osteopontin," Critical Reviews in Oral Biology and Medicine, vol. 11, no. 3, pp. 279-303, 2000.

[16] G. S. Stein, J. B. Lian, J. L. Stein, A. J. Van Wijnen, and M. Montecino, "Transcriptional control of osteoblast growth and differentiation," Physiological Reviews, vol. 76, no. 2, pp. 593629, 1996.

[17] M.-H. Lee, A. Javed, H.-J. Kim et al., "Transient upregulation of CBFA1 in response to bone morphogenetic protein-2 and transforming growth factor $\beta 1$ in $\mathrm{C} 2 \mathrm{C} 12$ myogenic cells coincides with suppression of the myogenic phenotype but is not sufficient for osteoblast differentiation," Journal of Cellular Biochemistry, vol. 73, no. 1, pp. 114-125, 1999.

[18] K. Miyama, G. Yamada, T. S. Yamamoto et al., "A BMP-inducible gene, Dlx5, regulates osteoblast differentiation and mesoderm induction," Developmental Biology, vol. 208, no. 1, pp. 123-133, 1999.

[19] K.-S. Lee, H.-J. Kim, Q.-L. Li et al., "Runx2 is a common target of transforming growth factor $\beta 1$ and bone morphogenetic protein 2, and cooperation between Runx2 and Smad5 induces osteoblast-specific gene expression in the pluripotent mesenchymal precursor cell line C2C12," Molecular and Cellular Biology, vol. 20, no. 23, pp. 8783-8792, 2000.

[20] K. Nakashima, X. Zhou, G. Kunkel et al., “The novel zinc fingercontaining transcription factor Osterix is required for osteoblast differentiation and bone formation," Cell, vol. 108, no. 1, pp. 1729, 2002.

[21] M.-H. Lee, Y.-J. Kim, H.-J. Kim et al., "BMP-2-induced Runx2 expression is mediated by Dlx5, and TGF- $\beta 1$ opposes the BMP2-induced osteoblast differentiation by suppression of Dlx 5 expression," Journal of Biological Chemistry, vol. 278, no. 36, pp. 34387-34394, 2003.

[22] K. Felber, P. M. Elks, M. Lecca, and H. H. Roehl, "Expression of osterix is regulated by FGF and Wnt $/ \beta$-catenin signalling during osteoblast differentiation," PLoS ONE, vol. 10, no. 12, Article ID e0144982, 2015.

[23] R. Matsumoto, T. Uemura, Z. Xu, I. Yamaguchi, T. Ikoma, and J. Tanaka, "Rapid oriented fibril formation of fish scale collagen facilitates early osteoblastic differentiation of human mesenchymal stem cells," Journal of Biomedical Materials Research A, vol. 103, no. 8, pp. 2531-2539, 2015.

[24] Y. Hayashi, T. Ikeda, S. Yamada, Z. Koyama, and K. Yanagiguchi, "The application of fish collagen to the dental and hard tissue regenerative medicine," in Seafood Processing By-Products: Trends and Applications, S.-K. Kim, Ed., chapter 22, pp. 455462, Springer, New York, NY, USA, 2014. 

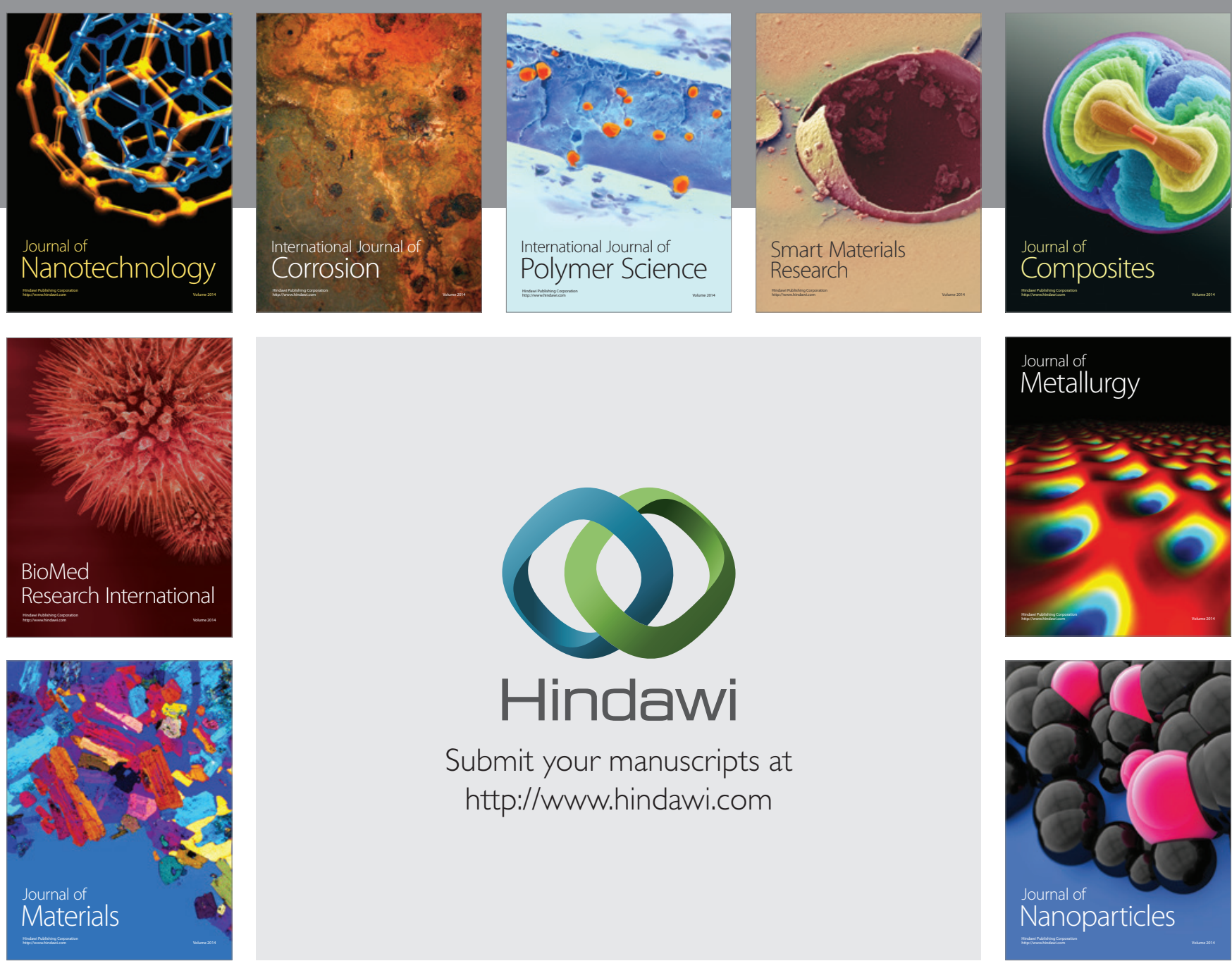

\section{Hindawi}

Submit your manuscripts at

http://www.hindawi.com

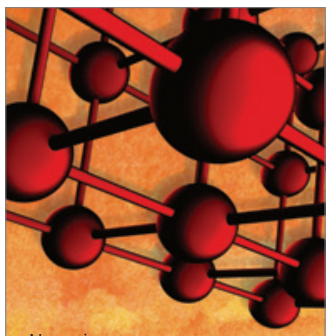

Materials Science and Engineering
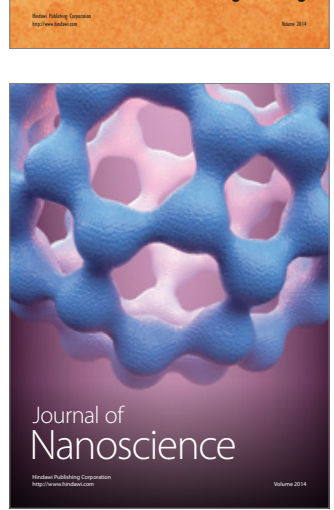
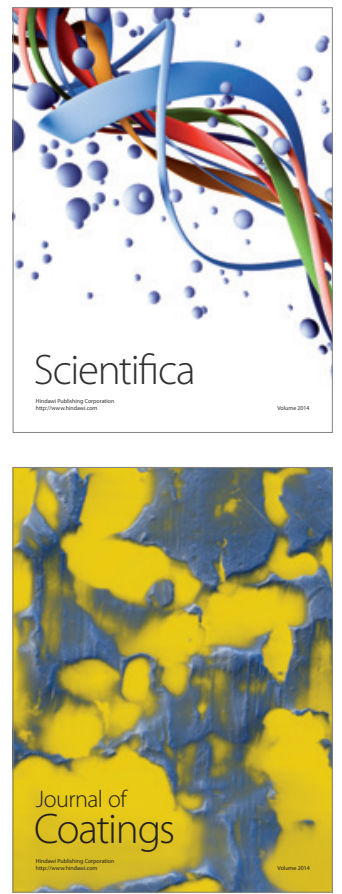
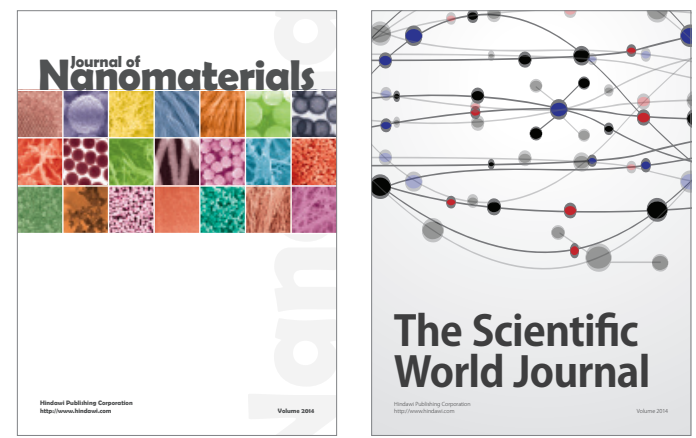

The Scientific World Journal
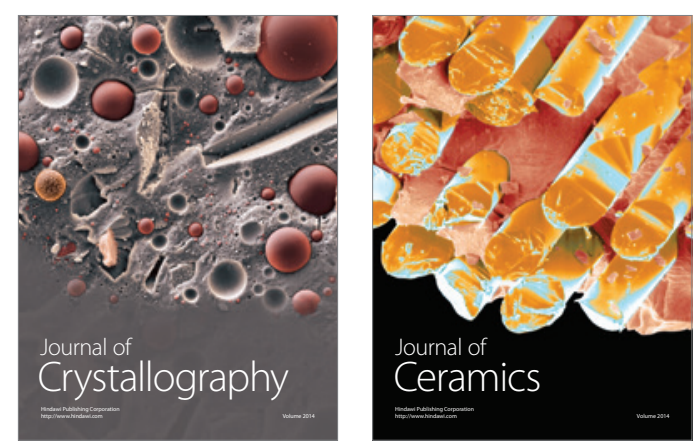
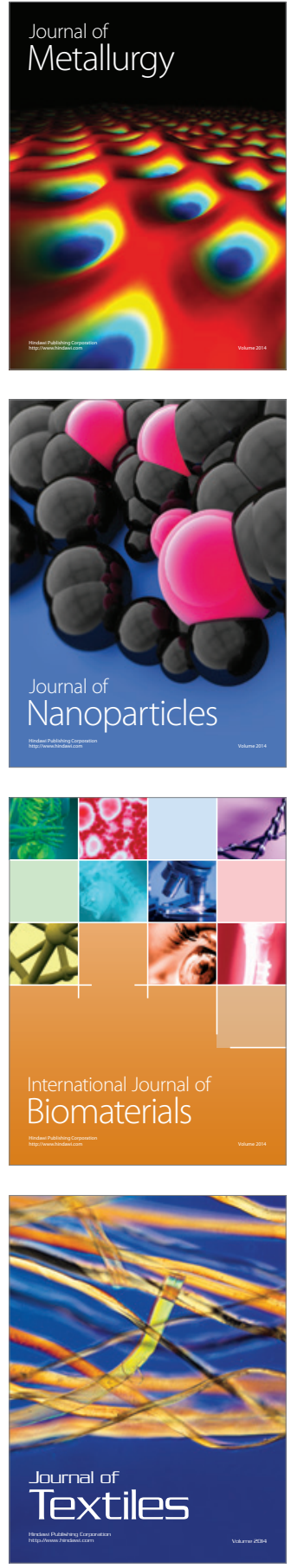